This item was submitted to Loughborough's Research Repository by the author.

Items in Figshare are protected by copyright, with all rights reserved, unless otherwise indicated.

\title{
Modelling the effect of spray breakup, coalescence and evaporation on vehicle surface contamination dynamics
}

PLEASE CITE THE PUBLISHED VERSION

https://doi.org/10.4271/2018-01-0705

PUBLISHER

(C) SAE International

VERSION

AM (Accepted Manuscript)

\section{PUBLISHER STATEMENT}

This work is made available according to the conditions of the Creative Commons Attribution-NonCommercialNoDerivatives 4.0 International (CC BY-NC-ND 4.0) licence. Full details of this licence are available at: https://creativecommons.org/licenses/by-nc-nd/4.0/

\section{LICENCE}

CC BY-NC-ND 4.0

\section{REPOSITORY RECORD}

Kabanovs, Anton, Andrew Garmory, Martin Passmore, and Adrian P. Gaylard. 2018. "Modelling the Effect of Spray Breakup, Coalescence and Evaporation on Vehicle Surface Contamination Dynamics". figshare. https://hdl.handle.net/2134/34307. 


\title{
Modelling the Effect of Spray Breakup, Coalescence and Evaporation on Vehicle Surface Contamination Dynamics
}

\author{
Author, co-author (Do NOT enter this information. It will be pulled from participant tab in \\ MyTechZone) \\ Affiliation (Do NOT enter this information. It will be pulled from participant tab in MyTechZone)
}

\begin{abstract}
Vehicle surface contamination is an important design consideration as it affects drivers' vision and the performance of on board camera and sensor systems. Previous work has shown that eddy resolving methods are able to accurately capture the flow field and particle transport, leading to good agreement for vehicle soiling with experiments. What is less clear is whether the secondary break-up, coalescence and evaporation of liquid particles play an important role in spray dynamics. The work reported here attempts to answer this and also give an idea of the computational cost associated with these extra physics models. A quarter scale generic SUV model is used as a test case in which the continuous phase is solved using the SpalartAllmaras IDDES model. The dispersed phase is computed concurrently with the continuous phase using the Lagrangian approach. The TAB secondary break-up and the stochastic O'Rourke coalescence models are used. The spray's rate of evaporation is calculated based on the relative humidity encountered on a typical October day in Britain. The secondary break-up model is found to be redundant, possibly due to the properties of spray. The coalescence model predicts high coalescence of particles close to the source and improves agreement with experiment, although at a high computational cost. Including evaporation removes small particles from the simulation and reduces overall contamination. When used along the coalescence model, evaporation is found to be negligible as it does not influence large particles to the same extent as it affects small particles. This suggests that droplet physics models need to be considered together as they can have a strong effect on each other as well as vehicle soiling. Here, we show that coalescence can be accounted for by using the time-averaged spray, obtained outside the region of high coalescence. This gives a very good agreement with experiment.
\end{abstract}

\section{Introduction}

Management of surface contamination is becoming an increasingly important research area and design criterion for all major vehicle manufacturers. Apart from aesthetic considerations, vehicle soiling can also affect customer satisfaction in instances when dirt gets transported to their hands and clothes upon the contact with the vehicle exterior [1]. Contamination of rear surfaces represents a particular inconvenience, as dirt accumulating on these surfaces can degrade visibility through the rear screen, reduce rear wiper life, deteriorate the performance by lighting and rear view camera systems, as well as reduce visibility of rear lamps to other road users.
With further development in advanced driver assistance systems and autonomous vehicles that rely on sensor and camera systems that must be kept clear, the ability to control surface contamination is expected to increase in importance [2], [3]. The source primarily responsible for rear surface contamination is referred to as selfsoiling. This happens due to spray generated by the rotation of the vehicle's own wheels, which pick up water and soil and the wheel wakes then advect them inwards, where they are picked up by the vehicle wake and are transported onto the rear surfaces of the vehicle. This is particularly problematic for square backed vehicle types, such as hatchbacks, estates and Sports Utility Vehicles (SUV) because the blunt rear geometry of these vehicles causes the formation of strong large-scale recirculating vortices that draw spray towards the rear surfaces [4]. Unlike rain droplets, spray generated by wheels can contain a diverse range of solid contaminants, such as soil, salt, sand, components of fuels and oils, carbon from combustion sources, brake and tyre wear and tarmac [1]. These materials, especially salt used for de-icing of roads, in combination with water can corrode the metalwork [5].

The ability to accurately predict surface contamination using numerical techniques would allow designers control or even resolve soiling issues early in a vehicle development programme, alongside the more usual areas of concern such as drag reduction, aeroacoustics and cross-wind stability. One of the earliest published Computational Fluid Dynamics (CFD) studies that looked into side and rear soiling of an SUV was reported by Yoshida et al. [6]. The geometry used was greatly simplified due to the limited computing capabilities of that time. The work used two methods for particle tracking: Lagrangian and Eulerian. An important conclusion was that the time averaged flow data can be used to predict vehicle soiling, but this has been subsequently refuted. For example, Reynolds-Averaged Navier Stokes (RANS) equations were seen to be unable to predict the soiling of the rear of a square back vehicle in [7], and the work of Paschkewitz [8], [9] demonstrated that the vertical distribution of particles in the wake of commercial vehicles is significantly different in steady-state and unsteady flow fields. This was also noted in the work of Jilesen et al. [10], who complemented the study of Gaylard and Duncan [4]. Unlike Gaylard and Duncan [4], who performed a study on side and rear surface soiling by tracking Lagrangian particles in a post-processing step for a number of frozen transient data frames, Jilesen et al. [10] used a fully transient particle tracking approach. This resulted in better agreement with the experiment, justifying that particles must be tracked concurrently with the flow field. In both studies, particles were emitted from the rotating wheels using the technique proposed by Kuthada and Cyr [11], and the size of particles was a constant diameter of $0.2 \mathrm{~mm}$. The work of

Page 1 of 12 
Kabanovs et al. [12] gave a further insight into the role of flow unsteadiness in vehicle soiling. It showed that large scale flow unsteadiness plays a crucial role as it affects particle entrainment into as well as dispersion within the wake. It was therefore found to be necessary to model the full time history of the particle paths rather than using a statistically averaged description of the turbulent dispersion. It has been shown that eddy resolving methods are required to accurately predict the flow field around bluff bodies and produce encouraging results of base soiling [13], as well as simulate the soiling trends with changes to vehicle geometry [14].

While most studies ([6], [8], [9], [4]) used one-way momentum coupling and did not take into account the influence of particles on the continuous phase, Gaylard et al. [15] showed that two-way momentum coupling can be important in some cases. Apart from flow field modification due to the presence of moving particles, particle-particle interactions (four-way coupling) may need to be considered for very dense sprays. These interactions can involve hydrodynamic coupling and particle inter-collisions. Hydrodynamic coupling refers to the force between a particle and the surrounding fluid, resulting in particle secondary break-up, while particle collisions can lead to coalescence and breakup [16]. This, along with evaporation, can affect the property of spray and its dynamics. One of the few CFD studies that considered both the break-up and coalescence of particles, as well as the back-coupling between the continuous and dispersed phases is the work by Hu et al. [17]. However, it discussed neither the computational cost of these models, nor provided any statistical or analytical information about the influence of the extra physics on the overall contamination results.

Kabanovs et al. [14] and [12], demonstrate broadly good correlation between experiments and CFD for base contamination, however the numerical results showed a narrow area of high soiling stretching along the edge of the base, not present in the experimental data. This was proposed to be due to a large fraction of small, low-inertia particles in the numerical spray. It was suggested that in the experiment these small droplets never reach the base because they either evaporate or coalesce into larger droplets. The current paper aims to test this hypothesis and understand whether secondary breakup and coalescence may be important in vehicle soiling studies. It also considers liquid evaporation. While evaporation is usually limited (relative humidity can be controlled and set as high as $95 \%$ [18]) in soiling experiments performed in climatic wind tunnels, the effect that evaporation has in on-road conditions, and how this influences the correlation between experimental and real-world situations, is unclear. Therefore, evaporation effects are also included in the study. These extra physics models are used both in isolation and in combination and their effect on predicted soiling patterns as well as the additional computational cost of their use is considered.

\section{Vehicle Model}

A quarter scale generic SUV model is used as a test case. It was designed within the department of Aeronautical and Automotive Engineering at Loughborough University and is representative of a typical SUV. The wheels are fixed and four pins support the model. The model can be configured to have different ride heights and rear geometry. In this study, a single configuration was used, shown in Figure 1. The ride height, the roof taper and diffuser were set to 0.065 $\mathrm{m}, 0$ degrees and 30 degrees, respectively. More information can be found in [19].
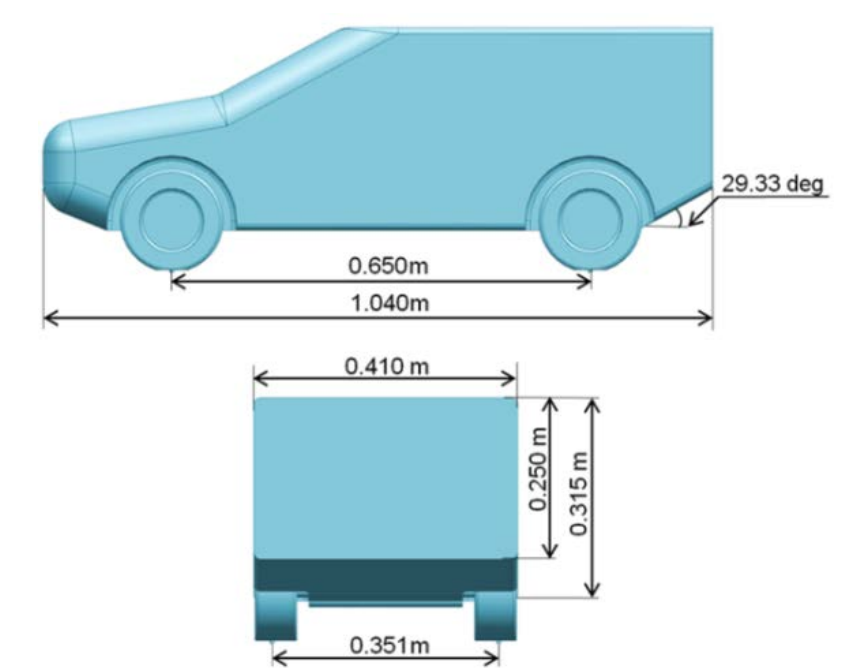

Figure 1. Generic SUV model.

\section{Experimental Methodology}

Full details of the experimental approach can be found in Kabanovs et al. [14]. However, the most important details are summarised here. The experiments were performed in the wind tunnel at Loughborough University. The wind tunnel has an open loop, circuit, closed working section configuration, with $2.53 \mathrm{~m}^{2}$ cross sectional area of the test section. This produces a blockage ratio of $5.58 \%$ when the quarter scale generic SUV model is used. All tests were performed at a freestream velocity of $40 \mathrm{~m} / \mathrm{s}$, giving a Reynolds number of 2.77 million based on the model length. The freestream turbulence intensity is highly dependent on the physical condition of the wind tunnel and the original value was determined to be $0.2 \%$. Full details of the wind tunnel can be found in [20].

A single nozzle, installed $0.086 \mathrm{~m}$ behind the centre of the left rear pin, was used in soiling tests. The nozzle was directed 45 degrees downstream to reasonably represent tyre spray. A single nozzle was used rather than one located behind each rear wheel because such an approach simplifies the analysis and improves comprehension of results. A mixture of de-ionised water and Ultra Violet (UV) dye (Uvitex at $0.03 \%$ concentration) was used as the contaminant, injected at $11 \mathrm{MPa}$ and giving a mass flow rate of $\dot{m}=3.48 \mathrm{~g} / \mathrm{s}$. The spray properties close to the nozzle were measured by running a separate Phase Doppler Anemometry (PDA) test. The PDA data were acquired in a Stokes flow and droplets were sampled at a multiple traverse points $10 \mathrm{~mm}$ away from the nozzle. Full details are available in Kabanovs et al. [14].

The model was run in the wind tunnel for a short period of time to allow the flow field to settle, after which a 30 second injection of the contaminant was made. Following every test, the UV lamp and a Digital Single-Lens Reflex (DSLR) camera were placed in the test section and an image of the soiled rear-surface captured. Intensity plots were later produced based on the fluorescence captured in the photographs. High intensity corresponds to high fluorescence, indicating the presence of thick liquid film on a surface. 


\section{Computational Methodology}

\section{Computational Mesh and Boundary Conditions}

The computational mesh was generated using the snappyHexMesh meshing utility, available within the OpenFOAM (version 2.4.0) [21] package, which is an open-source CFD tool. The mesh predominantly contains hexahedra and split-hexahedra cells. The meshing approach was very similar to the one used in Kabanovs et al. [14], and is shown in Figure 2. There are three sections that form a $28.0 \mathrm{~m} \mathrm{x} 1.92 \mathrm{~m} \mathrm{x}$ $1.32 \mathrm{~m}$ large computational domain. The outer sections have a slip wall boundary condition, while the middle (9.08 m x $1.92 \mathrm{~m} \mathrm{x} 1.32$ $\mathrm{m})$ contains the physical model and represents the measurement section with all walls set to no-slip condition, thus allowing the boundary layer to develop. It has divergent walls to match the physical wind tunnel arrangement and ensure that the longitudinal pressure gradient is zero when the domain is empty. Prism layers, with the appropriate thickness for a high $\mathrm{y}^{+}$are used around the model. A hybrid all-y+ wall model [22] is used at the walls to model the inner part of the turbulent boundary layer. The mesh contains $\sim 63$ million cells.
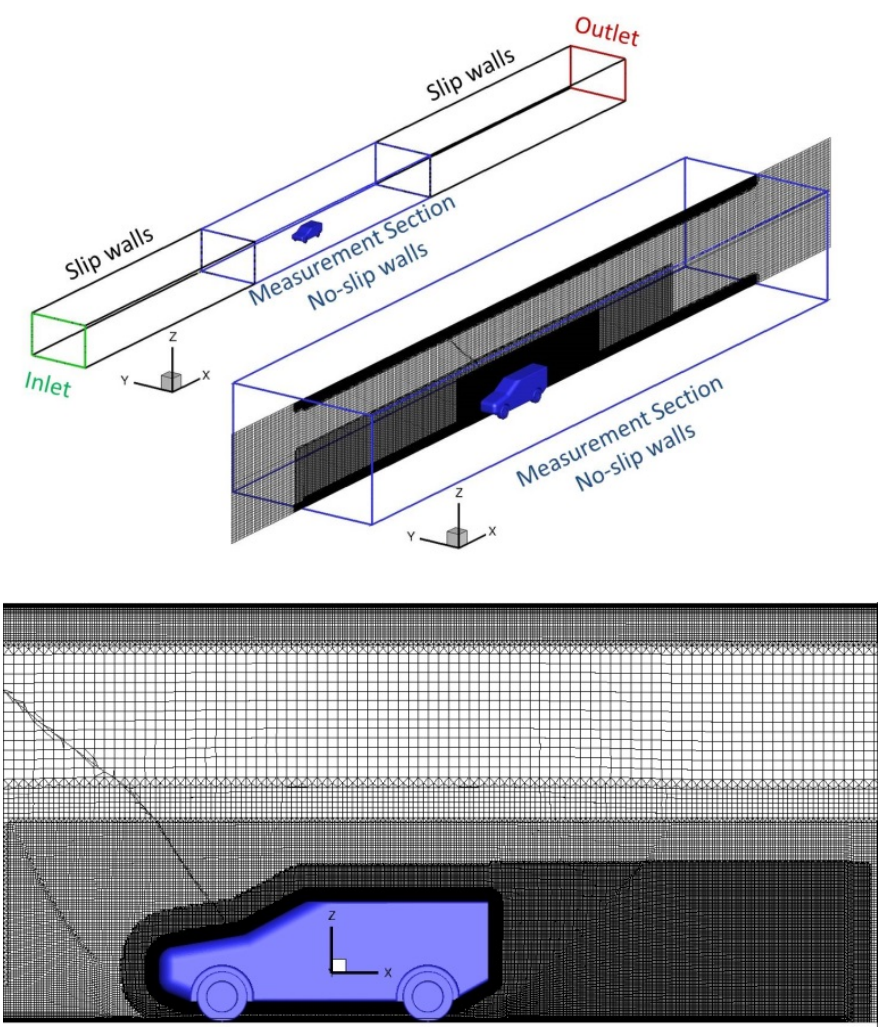

Figure 2. Computational domain and mesh around the generic SUV model.

\section{Modelling of the Continuous Phase}

OpenFOAM (version 2.4.0) [21] was used to run numerical simulations. The Improved Delayed Detached Eddy Simulation (IDDES) [23] was used that treats the boundary layer using a RANS method and applies the Large Eddy Simulation (LES) technique to separated regions. This greatly reduces the computational cost as near-wall models can be used to model the boundary layer, thus reducing the number of cells required in this region. In this work, the Page 3 of 12
RANS part of the IDDES used the Spalart-Allmaras (SA) turbulence model [24], which is formulated based on a single equation to derive eddy viscosity. The velocity profile at the walls was modelled using the all-y+ formulation, suggested by Spalding [22]. This is a hybrid formulation that includes the viscous sub-layer, buffer layer and the log layer, allowing a wider range of wall-normal computational grid spacing.

The Navier-Stokes equations were solved using the incompressible Pressure Implicit with Splitting of Operators (PISO) algorithm. A constant computational time step ( $\Delta t=4 \times 10^{-5} \mathrm{sec}$ ) was used throughout the simulation, which was around two times larger than that used in the previous work [12]. This choice was made to offset the significant computational cost of the coalescence model. Nevertheless, this gave a mean Courant-Friedrichs-Lewy (CFL) number of 0.08 with $\mathrm{CFL}<1$ in most of the wake. The maximum CFL number was around 18 , located in regions of high flow acceleration, particularly in a $4 \mathrm{~mm}$ gap between the floor and the wheel base. However, this did not affect the stability of computations and is unlikely to affect the accuracy of base soiling simulations. Second order numerical schemes were used.

\section{Modelling the Motion of the Dispersed Phase}

The dispersed phase was modelled concurrently with the continuous phase using the Lagrangian approach [25] as recommended for accurate simulations in Kabanovs et al. [12]. The Lagrangian multiphase capability in OpenFOAM was used with in-house modifications made to include evaporation, coalescence and breakup. The Lagrangian approach allows tracking individual particles, although the concept of parcels is usually used to reduce computational cost. The numerical parcel represents a group of identical particles and has the property of the representative particle, as shown schematically in Figure 3. Although the increase in the number of parcel streams refines the spray as it creates a larger ensemble of tracks, it also increases computational cost. In this work, a total of 13.125 million parcels were tracked, released uniformly throughout the simulated time. All parcels carried the same total mass. To reduce computational cost, the parcels were removed from the domain when $0.8 \mathrm{~m}$ downstream from the base. As the length of flow recirculation at the rear of this SUV configuration had been identified to be around $0.3 \mathrm{~m}$ [12], this distance was deemed to be sufficient not to influence the results.

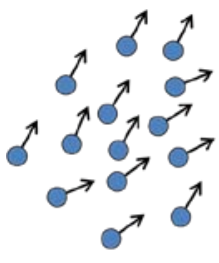

Individual particles

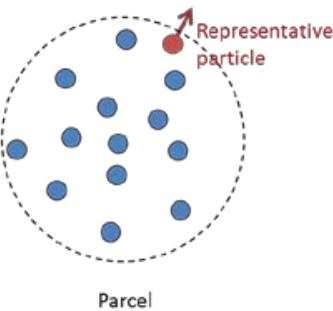

Parce
Figure 3: Schematic representation of the concept of numerical parcels.

Particle drag, gravity and shear lift are considered in the equation of motion, modelled as reported in Kabanovs et al. [12]. A stochastic dispersion model (Gosman and Ioannides [26]) is used to model the influence of the unresolved turbulent content on the motion of particles, also discussed in [12]. 


\section{Modelling Spray Evaporation}

In this work, a very simple one dimensional model, suggested by Spalding [27] and presented in Equation 7, was added to the existing OpenFOAM spray modelling to estimate the rate of liquid evaporation. This formulation considers isolated and spherical droplets that are evaporating in an infinite environment. Current work assumes constant temperature evaporation and does not take into account energy conservation and mass that has evaporated within a time-step is removed from the simulation. This significantly simplifies the simulation and allows the model to be incorporated into the incompressible solver in OpenFOAM and the simplifications reduce the computational cost of the evaporation model. The rate of evaporation depends on the particle size, which is recalculated after each time step based on the new mass, which is $m_{p, n}=m_{p}-m_{p \text {,evap }}$. Here, the $m_{p}$ is the particle mass in the beginning of the time step, $m_{p, \text { evap }}$ is the mass that has evaporated within a time step and $m_{p, n}$ is the new particle mass.

$$
\frac{m_{p, e v a p}}{\Delta t}=\pi D_{v} \operatorname{Sh} \rho D \ln \left(1+B_{M}\right)
$$

In Equation 7, $\Delta t$ is the time step, $D_{v}$ is the diffusion coefficient, Sh is the Sherwood number, $\rho$ is air density, $D$ is the particle diameter and $B_{M}$ is the Spalding's mass transfer number. The Sherwood number represents the ratio of convective mass transfer to the rate of diffusive mass transport and is expressed using the Ranz \& Marshall correlation [28], shown in Equation 8. This correlation takes into account the effect of particle slip velocity $U_{\text {rel }}$, expressed via the particle Reynolds number $R e_{p}$, which represents the ratio of inertial forces to the viscous forces of flow around a particle and is given in Equation 9. The Schmidt number $S c$ is given in Equation 10 and is defined as the ratio of momentum diffusivity and mass diffusivity.

$$
\begin{gathered}
S h=2+0.55 R e_{p}{ }^{0.5} S c^{0.33} \\
R e_{p}=\frac{\rho\left|U_{r e l}\right| D}{\mu} \\
S c=\frac{\mu}{\rho D_{v}}
\end{gathered}
$$

The dynamic viscosity $\mu$ and the diffusion coefficient $D_{v}$ are chosen according to the temperature of the dispersed phase, which is kept constant throughout the whole simulation. The ambient temperature stays constant as well. Finally, the Spalding's mass transfer number used in Equation 7 is defined in Equation 11.

$$
B_{M}=\frac{Y_{F, S}-Y_{F, \infty}}{1-Y_{F, S}}
$$

Here, $Y_{F, s}$ and $Y_{F, \infty}$ are the vapour mass fractions at the particle surface and in the far field, respectively. This work assumes that the vapour at the droplet surface is always saturated (relative humidity $=$ $100 \%$ ), resulting in an instant and steady evaporation. The vapour mass fractions $Y_{F, s}$ and $Y_{F, \infty}$ can be calculated using the humidity ratio $w$, which is a mass based ratio between water and dry air. It can be obtained from a psychrometric chart, provided that the relative humidity and the dry bulb temperature are known. Then, the $Y_{F, s}$ and $Y_{F, \infty}$ can be calculated using Equation 12.

$$
Y=\frac{w}{1+w}
$$

In reality, $D_{v}, Y_{F, s}$ and $Y_{F, \infty}$ are temperature dependent but in the scope of this work are assumed to be constant during the evaporation process. Therefore, the vapour mass fractions depend only on the humidity and temperature, which are not affected by evaporation.

Although the evaporation model used in this study is simplified, it still represents the mechanism and allows investigation into how it would affect surface contamination. In this work, the relative humidity in the free stream was chosen to be $84 \%$, which is the mean value in Britain in October [29]. The wet bulb temperature at particle surface was chosen to be of room temperature (292 K), as the water was stored in a tank in the balance room, prior to spraying it in the wind tunnel. The dry bulb temperature of the free stream was 289.5 $\mathrm{K}$, as measured on the day of experiments. It should be stressed that the humidity ratio, defined by the relative humidity and the bulb temperature dictates how much liquid will evaporate and any uncertainty will lead to incorrect results. It can be seen in Figure 4 that the difference in the relative humidity of only $5 \%$, as well as the difference in dry bulb temperature of water of $2.5 \mathrm{~K}$ can affect the evaporation of spray significantly. This figure also shows the importance of initial droplet size on evaporation time. Since the exact value of the relative humidity and temperatures on the day of experiments is unknown, the absolute accuracy of the model is less relevant than the general trends observed.

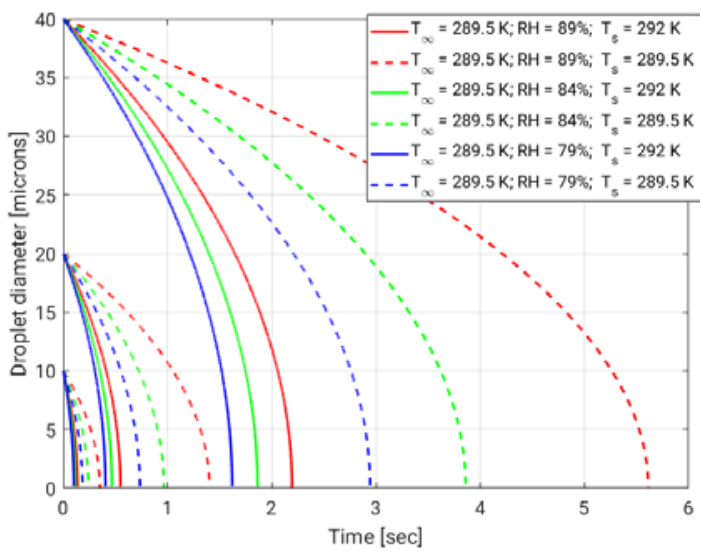

Figure 4: The rate of evaporation of particles with different ambient relative humidity (RH).

\section{Modelling Spray Coalescence}

The coalescence of particles induced by collision can have a significant influence on the average particle size in the spray. The stochastic particle collision algorithm proposed by O'Rourke [30] is one of the most widely used models for calculating coalescence in simulations with the Lagrangian particles and is available in OpenFOAM. Details are provided here for reference. When the concept of parcels is used, the model assumes that two parcels can collide if they are located in the same computational cell. This is the primary condition imposed by the model that needs to be satisfied. The probability that parcels undergo either grazing collision (bouncing) or coalescence is then calculated as a function of particle diameters, relative velocity and cell volume. Frequently, the parcels that are most likely to collide are those located on the opposite cell boundaries and are moving away from each other, as these have the largest relative velocity and are yet in the same computational cell. Since the outcome of collision is either elastic bouncing or coalescence, the trajectories of parcels near cell boundaries are preferentially eliminated, explaining the dependency of the model to

Page 4 of 12 
grid resolution. The dependency of the O'Rourke collision model on the computational mesh is discussed in greater detail in ([31] [32], [33]). For grazing collision, the parcel speed and direction after collision is computed using the random restitution coefficient, ensuring that the momentum is conserved. In the event of coalescence a random number of particles are transferred from the smaller parcel into the bigger one. The size of particles in the second parcel is then calculated taking into account the added mass due to coalescence. Figure 5 presents a schematic of the O'Rourke coalescence model. The new velocity of the parcel which has increased in mass is calculated based on conservation of momentum.

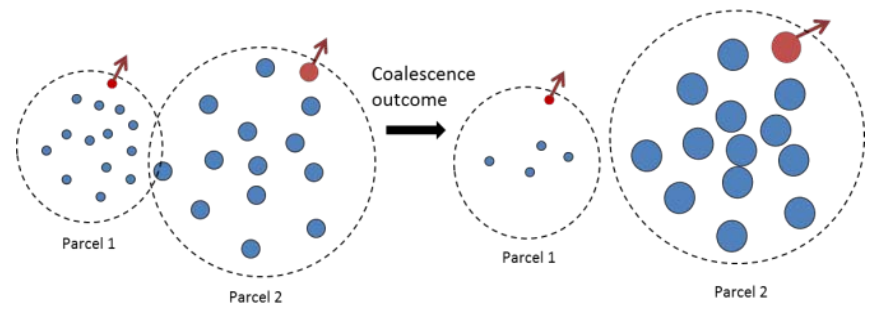

Figure 5: Schematic representation of the O'Rourke coalescence model.

The original O'Rourke model implemented in OpenFOAM can only be used in a compressible solver because it was developed to be used primarily in combustion simulations. To modify the model for an incompressible solver, the energy conservation algorithm was removed and the temperature dependent variables were set constant. The model has also been extended to avoid the number of particles in a parcel reducing to a value of less than one. When such a case happens, all mass is transferred to the second parcel and the first parcel is removed from simulation.

\section{Modelling Spray Break-up}

In this work, the secondary break-up (as distinct from the primary breakup that occurs when the initial spray is formed) is modelled using the Taylor Analogy Breakup (TAB) model, suggested by O'Rourke et al. [34]. The model is based on the analogy between an oscillating and distorting particle and a spring-mass system. The aerodynamic force represents the external force applied to a springmass system while the liquid viscosity represents the damping force. The formulation of this model is such that there is not a unique critical Weber number, which is a dimensionless number that represents the ratio of inertia forces to the surface tension of a particle and is often used to characterise particle break-up conditions. Instead, the break-up event depends on the history of particle slip velocity, which leads to oscillation and displacement of the equator of the particle from its equilibrium position. Larger particles are influenced by this to a greater extent as oscillations of small particles are easily damped. The break-up occurs only if the amplitude of oscillations normal to the slip velocity vector reaches the particle radius. The major drawback of the model is that it only tracks one oscillation mode, while there can be multiple modes present. In the event of break-up, the size of product particles in a parcel is calculated based on the surface energy, assuming that the energy of the parent particle before break-up is equal to the sum of energies of the product particles after break-up. The mechanism is illustrated in Figure 6. For more details of the model and its numerical implementation readers are referred to [34]. In this work, the model was adjusted for use in an incompressible solver. The dependency of gas-liquid surface tension coefficient $\sigma$ on the ambient temperature was ignored and $\sigma$ was set to a constant value.

Page 5 of 12

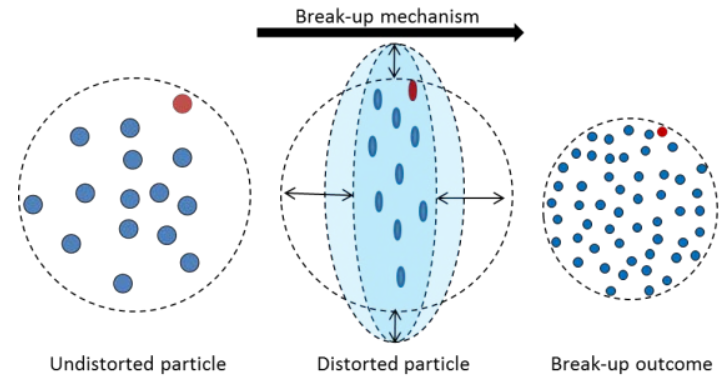

Figure 6: Schematic representation of the TAB break-up model.

\section{Test Cases}

Three test cases were considered in this study. The primary test case included three Lagrangian "clouds", tracked concurrently with the flow field in a single simulation. There is no cloud-cloud interaction. Such an approach reduces the computational cost by eliminating the need to run many simulations separately. In addition, it also improves data comparison as it ensures that each cloud sees the same instantaneous flow field and any difference in the results is a function of cloud properties. On the other hand, the nature of such computational approach makes the modelling of the momentum back-coupling impossible and therefore was not considered in this study. The first cloud used the original spray, as measured previously close to the nozzle using PDA, and considered neither the evaporation, nor the coalescence and break-up of particles. The second cloud considered evaporation and the third cloud used the O'Rourke and TAB models, explained previously. The second simulation used a combination of all aforementioned mechanisms. As will be shown next, consideration of the coalescence mechanism has improved the numerical results, although at a very high computational cost. Therefore, the third simulation used the new spray obtained downstream of the nozzle in the simulation that used the O'Rourke model to see if this is sufficient to account for the influence of spray coalescence on the eventual soiling pattern. Table 1 presents all the cases considered.

Table 1: Computational cases.

\begin{tabular}{lcccc}
\hline & Cloud No. & Spray & Evaporation & $\begin{array}{c}\text { Collision } \\
\& \text { Break-up }\end{array}$ \\
\hline \multirow{2}{*}{ Simulation 1 } & 1 & Original & - & - \\
& 2 & Original & Yes & - \\
\hline Simulation 2 & 1 & Original & - & Yes \\
\hline Simulation 3 & 1 & Original & Yes & Yes \\
\hline
\end{tabular}

\section{Results}

\section{Computational Cost}

All simulations have been run on x86 nodes, each node incorporating $28 \mathrm{CPU}$ and 128 GB RAM. Initial single phase simulations were run to establish the flow field. These were run for 1 second of simulated time. The aerodynamics results are not shown here, but can be found in our previous work [12], which used the same configuration of the SUV model; good agreement was obtained between experiments and 
CFD in terms of base pressures and the flow field. The agreement obtained in the current work is very similar. The most computationally expensive model used in this work is the O'Rourke collision model. The high cost of this model is associated with the necessity to loop over all parcels in the domain and search for the pairs that have a non-zero probability of collision. Figure 7 presents results of a study, undertaken to understand the influence of the number of injected parcels on the computational cost of the coalescence model. In the study, a different number of parcels were injected into a frozen flow field within a single time step and the time taken to compute one step was recorded. The particles were injected into a single processor. Figure 7 shows that the computational cost of the O'Rourke model is very dependent on the number of parcels injected and the probability of collision. As can be seen, the computational cost increases rapidly with the number of parcels injected into the domain. In addition, Figure 7 shows that it takes a considerable amount of time to find all the pairs of parcels located in the same computational cell, regardless of their probability to collide.

In the soiling simulations, 350 parcels were injected at each time step, with the total number of parcels in the domain reaching $\sim 300,000$. As the parcels are injected at one location they are concentrated in a limited number of the processors used for the calculation. As such, the parallel efficiency is relatively poor for the dispersed phase in these calculations. This would have resulted in a very high computational cost unless the coalescence model was constrained to a limited region as was done after 0.2 seconds when the region where coalescence is important could be identified. Figure 8 shows the fraction of coalescence events in the first simulation (cloud 3), normalized against the maximum number in the whole domain. It also shows the region (black dashed line) to which the coalescence modelling was eventually constrained. As can be seen, the coalescence of particles appears to be taking place very close to the injector location, and therefore the coalescence model had been restricted in the wake and in the region considerably away from the source. Three soiling simulations, as shown in Table 1 were run for 1.5 seconds of simulated time each. The first simulation used three clouds and required around $729 \times 10^{3} \mathrm{CPU}$ hours of computational effort. The second simulation used only one cloud but still required $672 \times 10^{3} \mathrm{CPU}$ hours to compute the same time period. This is associated with the high computational cost of the coalescence model, used in these simulations. For comparison, the third simulation used two clouds and considered neither of the extra mechanisms, thus requiring only $37.7 \times 10^{3} \mathrm{CPU}$ hours to compute 1.5 seconds, thus showing the high cost of computing coalescence even when restricted to the near nozzle region.

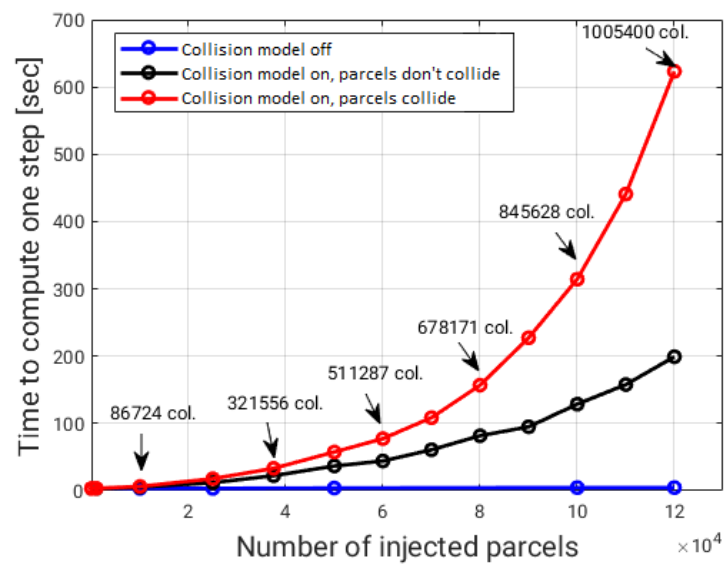

Figure 7: Computational cost of the coalescence model.

Page 6 of 12

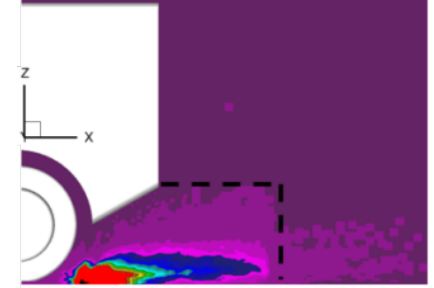

(a)

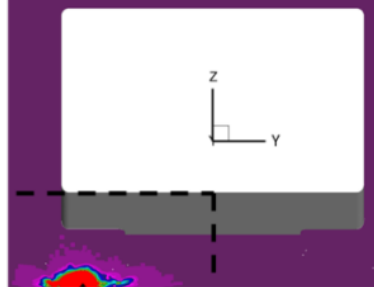

(b)

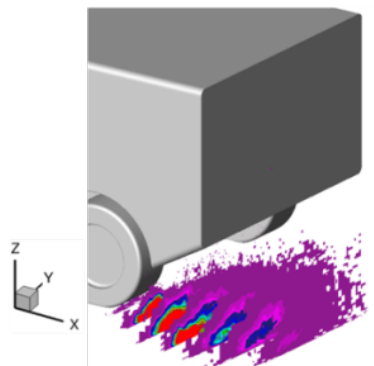

(c)

Collision Events [\%]: $\quad \begin{array}{lllllll}0 & 0.0004 & 0.0008 & 0.0012 & 0.0016 & 0.002 & 0.0024\end{array}$

Figure 8: Fraction of coalescence events, shown on $\mathrm{ZX}$ plane at $\mathrm{Y}=-0.1753$ $\mathrm{m}$ (a), ZY plane at $\mathrm{X}=-0.116 \mathrm{~m}$ (b) and on multiple ZY planes with $\mathrm{X}$ ranging from $-0.116 \mathrm{~m}$ to $0.15 \mathrm{~m}(\mathrm{c})$.

\section{Soiling Results Obtained in Simulations 1 \& 2}

Figure 9 shows the method used to map the deposited Lagrangian parcels onto a surface mesh and construct soiling intensity plots. This surface mesh is used for post-processing and is not the CFD mesh but is used to smooth the base soiling pattern. The parcels are first distributed around the grid cells by moving them to the closest cell node. The soiling intensity figure is then generated by normalising the mass of liquid that has accumulated in each cell node against the maximum value across all cells. The distance between grid points used in this study was $7 \mathrm{~mm}$.

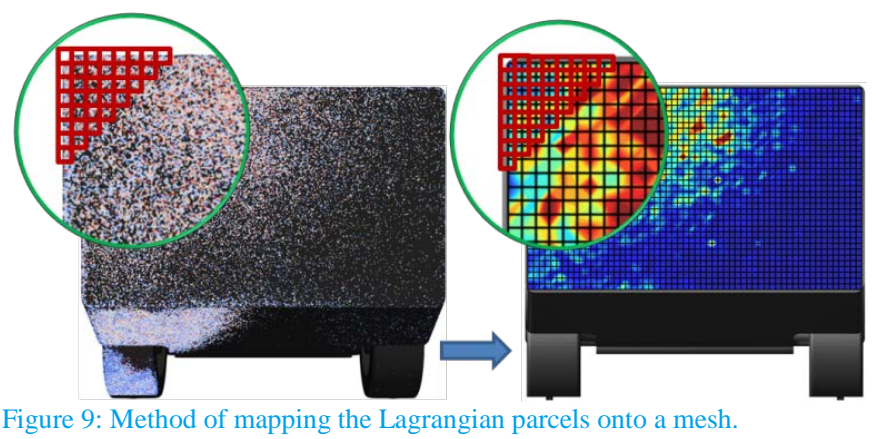

Figure 10 (a) shows the absolute soiling intensity collected during experiments, averaged over the same $7 \mathrm{~mm}$ grid while (b-e) present the computational results. The experimental plot is normalised by the highest experimental intensity. All simulated base soiling values have been normalised against the maximum value found in the baseline case (b). This allows qualitative comparison of soiling patterns between the numerical and experimental results but allows quantitative comparisons between baseline and modified simulations. Although (b) shows a very good agreement with the experiment shown in (a), an area of high contamination can be seen in the computational results stretching down the left edge of the base that is 
not present in the experimental data. The effect of evaporation and coalescence/breakup can be seen in (c) and (d) of Figure 10. The combination of all mechanisms is shown in Figure 10 (e). It should be pointed out that while a secondary break-up model was used no breakup events were predicted in all simulations, probably due to the properties of the spray used. This is interesting, because the overall size of particles appears to be too small for any secondary break-up to happen even when the coalescence is considered. This suggests that it was valid to ignore the break-up mechanism in the previous work [12], [14]. As can be seen, all three cases don’t show the highly contaminated area on the edge and therefore present improved agreement with the experimental soiling pattern. When evaporation alone is considered (Figure 10 (d)) a significant reduction in the soiling is observed compared to the baseline simulation (Figure 10 (b)). However, when evaporation is used at the same time as coalescence (Figure 10 (e)) very little difference is seen compared to coalescence alone (Figure 10 (c)). It is evident that the high contamination area at the edge of the base, seen in the baseline case, has been removed in all cases with extra physics models included. This is explored further in Figure 11 which shows the paths of all parcels deposited within a $5 \mathrm{~mm}$ distance from the left edge of the base during the final 0.125 seconds of computation. The results for two cases are compared: the baseline case and the case which considered all mechanisms together. It can be seen that the baseline case has a much larger proportion of small parcels (each carrying the same total mass) hitting the edge. These have been greatly reduced in the case with all physical mechanisms considered. Hence we can conclude that neglecting these processes leads to an over estimation of the number of small particles that are then deposited on the edge of the base in a way not observed in experiment.

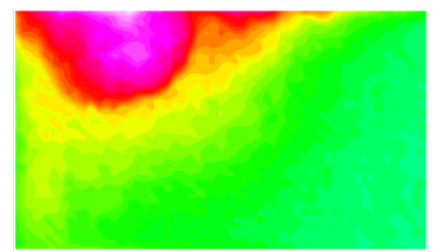

(a)

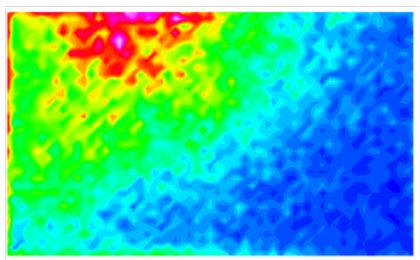

(b)

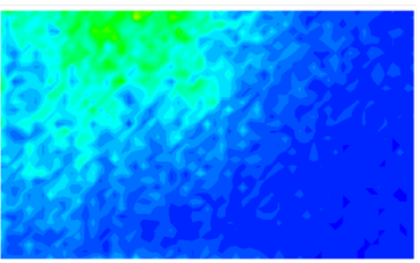

(d)

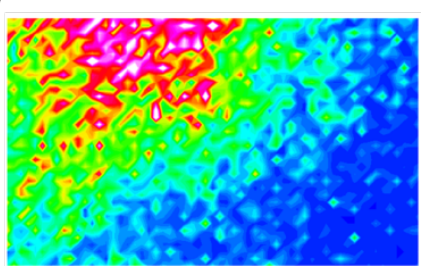

(c)

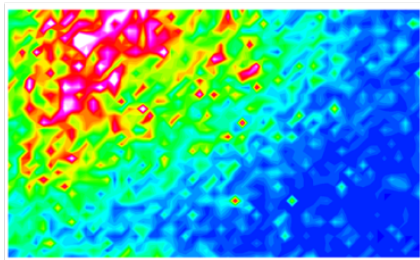

(e)

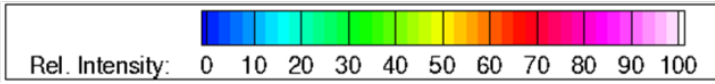

Figure 10: Contamination on the base: Experimental (a); Baseline (b); With coalescence and break-up (c); With evaporation (d); With coalescence, breakup and evaporation (e). Numerical results are normalised against the baseline case (b).

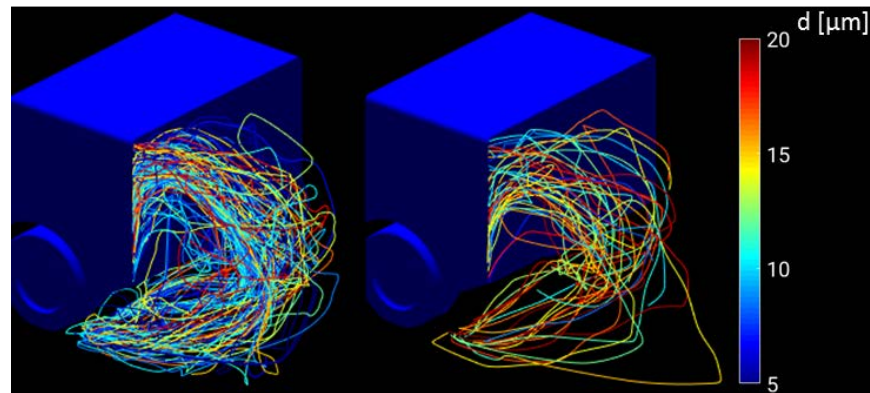

Figure 11: Paths of all parcels deposited within a $5 \mathrm{~mm}$ distance from the left edge of the base during the final 0.125 seconds of computation: Baseline left; with coalescence, break-up and evaporation right.

Figure 12 presents the mass-weighted size distribution of particles that have deposited on the base. The mass-weighted particle size distribution in the spray is also shown. This provides statistical information about the fraction of mass that is carried by particles of specific sizes. It is interesting that although the fraction of $3 \mu \mathrm{m}$ particles is the largest in the original spray, not many of them reach the rear surface in the baseline case. This preferential entrainment of large particles is further discussed in [12]. It can be seen that the evaporation model removes excessively small particles, which in turn leads to an increased fraction of larger (approximately $16 \mu \mathrm{m}$ ) particles that do not evaporate within the time required to reach the base. When the coalescence of particles is taken into account, the size distribution flattens a little and the particle peak size shifts to around $23 \mu \mathrm{m}$. The addition of the evaporation model does not make a big difference to the size distribution. This is due to coalescence happening very close to the source, forming large particles within a very short time after injection. These big particles have a much lower rate of evaporation.

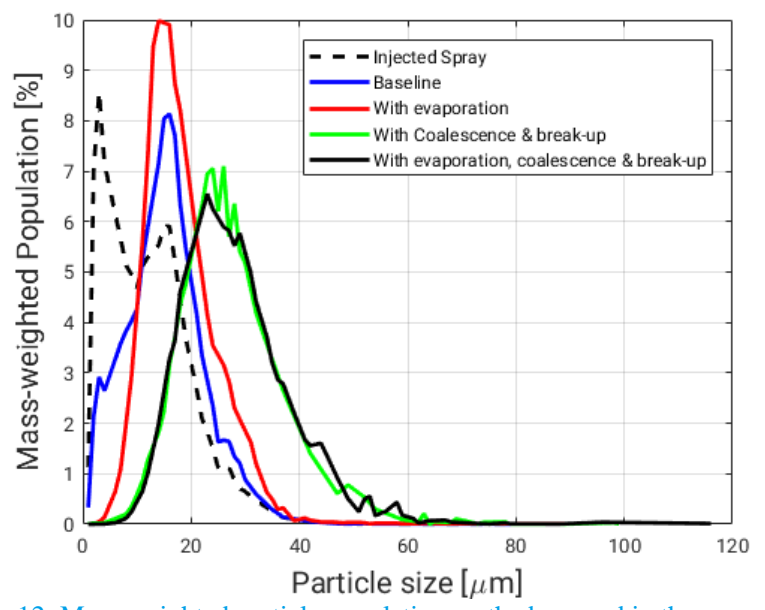

Figure 12: Mass-weighted particle population on the base and in the spray.

The difference in the amount of evaporated liquid per time step between two cases, both of which considered evaporation can be studied in Figure 13. It can be seen that the amount of evaporated mass in the case that did not take into account particle coalescence is significantly larger. This is due to particle coalescence taking place within a very short time upon injection, as was shown in Figure 8. 


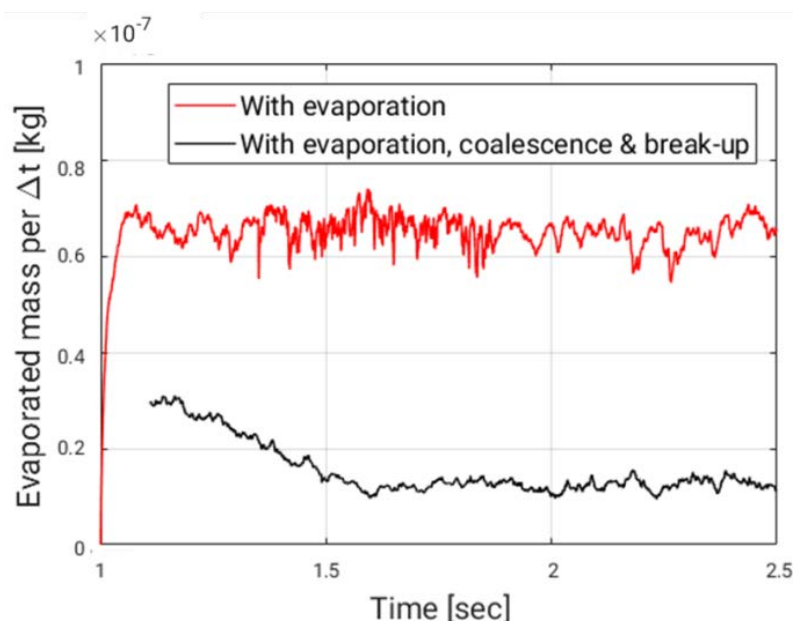

Figure 13: Rate of liquid evaporation.

Figure 14 shows the integrated numerical contamination evolution over time. The injection begins at 1 second and stops at 2.5 seconds. The first contamination event occurs at around 0.05 seconds after first injection. As can be seen, the total amount of mass on the base in the case that considered evaporation is almost three times smaller than that in the baseline case. As has been noted before, the evaporation barely changes the mass of particles in the simulation with the coalescence model present, leading to a very similar total contamination as obtained in the coalescence \& break-up case. These results show that the influence of evaporation on vehicle soiling is highly dependent on the other physical processes involved. For this case, the increased mean particle size caused by coalescence makes the evaporation have little effect.

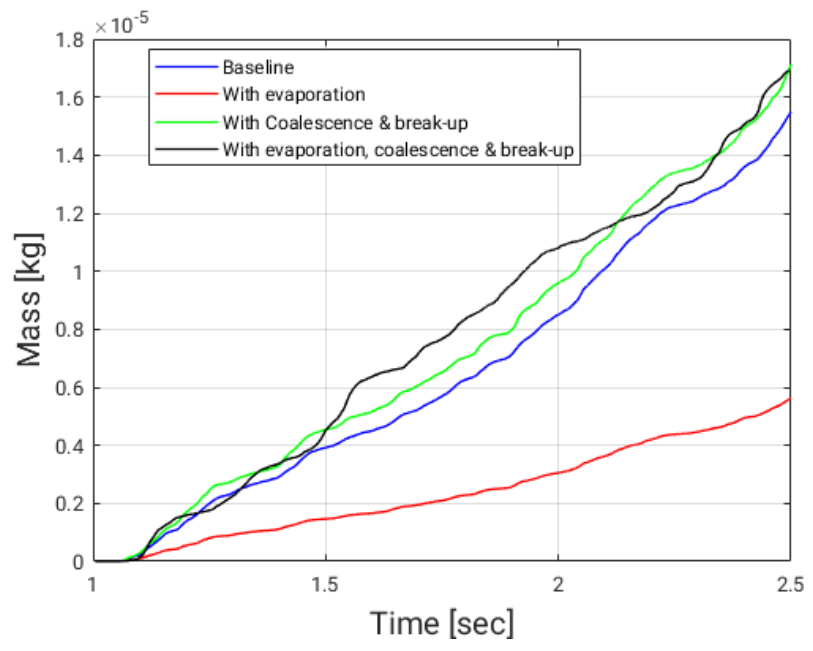

Figure 14: Numerical evolution of contamination.

A clear correlation between the dynamics within the soiling process shown in Figure 14 can be seen for the baseline case (blue) and the case that considered coalescence (green). This is because these were simulated within one simulation. The soiling process that considered all models together in a different simulation looks very similar, but the pulses of increased soiling rate are shifted. These pulses are associated with the mechanism of particle entrainment into the wake, which reveals unsteady, large scale and spatially correlated flow structures in the process [12].

\section{Soiling Results Obtained in Simulation 3}

It has been shown in the previous section that consideration of the coalescence for fine and dense sprays may be important. However, significant computational costs associated with models that can simulate this mechanism make it extremely expensive to use. The alternative to computing particle coalescence during the simulation would be to inject the fully developed spray formed in the dense spray region close to the nozzle, with the coalescence in the rest of the domain ignored. In this work, to obtain the properties of the fully developed spray, parcels were sampled at a frequency of $200 \mathrm{~Hz}$ in the volume located in the computational domain and indicated in red in Figure 15 (a). The data was then time-averaged. The sampling volume shown in Figure 15 (a) has a shape of a hollow quarter sphere, with a thickness of $0.02 \mathrm{~m}$. As can be seen, the radius of the quarter sphere is of an appropriate size to cover the region of high particle coalescence seen in this case. The sensitivity of the averaged spray properties computed in Simulation 1 cloud 3 (coalescence \& break-up case) to the position of the sampling volume was examined by changing the location of the original sampling volume and shifting it closer to the injection point as shown in Figure 15 (b) and (c). Figure 16 presents both spatial and temporal sensitivity of sampled data. It is evident that the statistics of spray sampled in all volumes are very similar. It can be also seen that sampling needs to be done for at least 1 second to obtain a well converged particle size distribution.
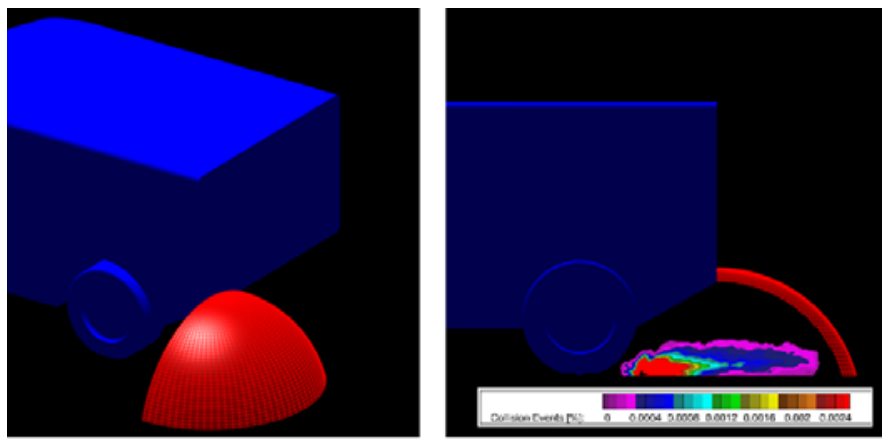

(a) Original sampling volume 1 .

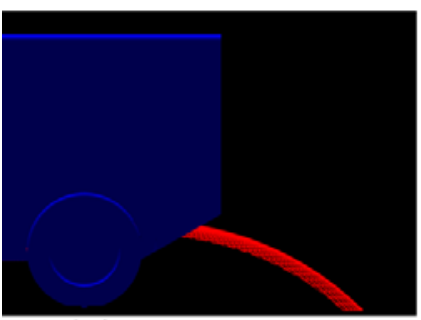

(b) Sampling volume 2 .

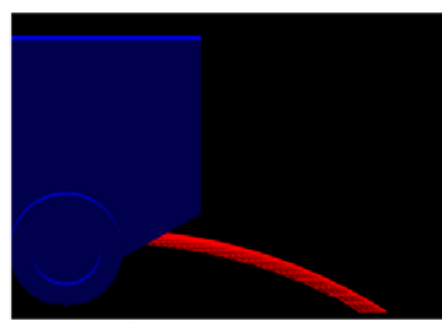

(c) Sampling volume 3.
Figure 15: Original sampling volume 1, used to obtain time-average particle size distribution. The region of high coalescence is also shown. (a); Additional volumes used to check sensitivity of the averaged spray properties to the position of sampling (sampling volume 2 (b) and sampling volume 3 (c)). 


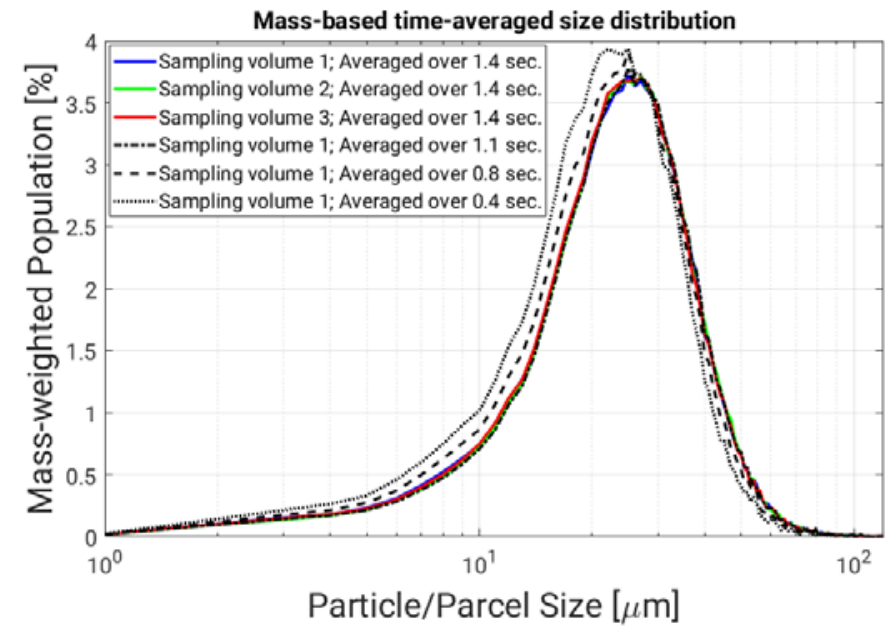

Figure 16: Spatial and temporal sensitivity of sampled data.

Figure 17 presents the time-averaged size distribution of particles based on the absolute mass in the sampling volume 1 , shown here for all computational cases considered. The data was averaged over 1.4 seconds. Here, the effect of each mechanism on the total mass carried by parcels of specific sizes can be studied. It can be seen that the most mass in the baseline case is transported by small particles, which evaporate rapidly when the evaporation model is used, leaving bigger particles in the spray. When the coalescence of particles is considered and evaporation is ignored, bigger particles are formed out of smaller ones, conserving the total mass. It is interesting to note that when the coalescence and evaporation are considered together, both the distribution and mass is very similar to the coalescence $\&$ break-up case with a difference only seen for the smallest particles. This is because evaporation has less effect on the larger particles.

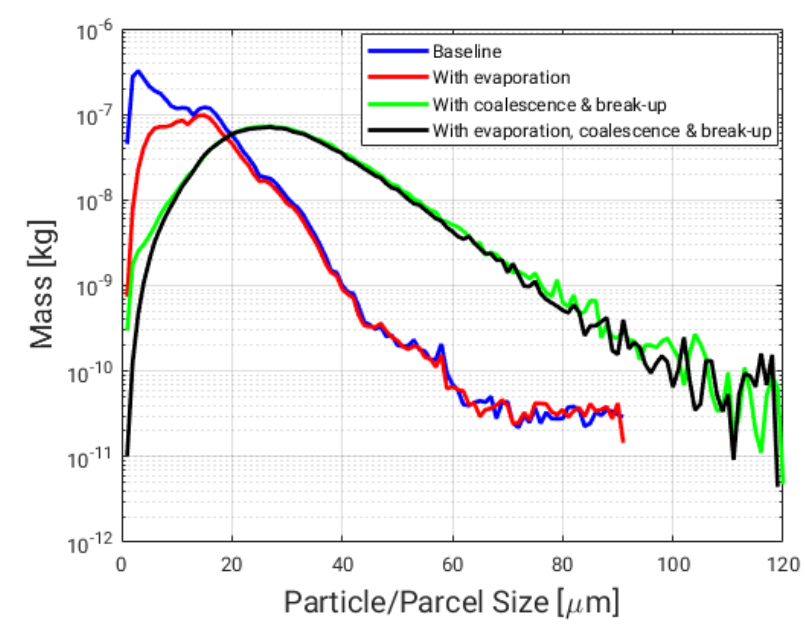

Figure 17: Mass-weighted (absolute) particle population in the sampling volume.

Spray sampled in volume 1 in simulation 1 (cloud 3 - coalescence \& break-up case) and averaged over 1.4 seconds (see Figure 16) was the "developed" spray used in the final simulation. This case was selected rather than the one that used all models together because the evaporation mechanism highly depends on the ambient conditions, unknown in this work. However, the difference in particle size distribution between these two cases is insignificant and is expected to give very similar soiling patterns. The evaporation model could be used with the fully developed spray injection at a relatively little cost in cases where this is important. The final simulation also uses the original spray to see the influence of particle size on the contamination results. The clouds do not influence each other and are injected from the same original location, with the same initial velocity. No additional physics models are used in either simulation and hence the computational cost is greatly reduced. Figure 18 (b) and (c) present the contamination patterns obtained with the original and new sprays, respectively. The intensities shown have been normalized against the original baseline case Figure 10 (b). The total mass deposited on the base in the baseline case in this simulation is approximately 1.1 times larger than that in simulation 1 . In addition, it can be seen that the contamination pattern has shifted towards the left edge and down. As can be seen in Figure 14 the soiling rate can be non-linear so the time at which soiling patterns are observed can affect instantaneous results. This further indicates the presence of unsteady modes within the soiling process, as discussed in [12], and suggests that soiling simulations on susceptible geometries must be run for a longer time period in order to be able to compare them against the qualitative and quantitative data collected in other numerical cases or experiments. The pattern obtained with the modified spray (c) shows better agreement with the experimental results than that with the original spray. The results are also seen to agree well with those obtained in the simulation using coalescence and evaporation modelling. It is evident that the region of high contamination is well captured, considering the short period of simulated time (only 1.5 seconds). It can be seen that the region of contamination stretching along the left edge of the base has also been removed. Finally, Figure 19 shows the spatial distribution of spray obtained in simulation 1 cloud 3 (coalescence \& break-up case) and simulation 3 cloud 2 (developed spray) on the interface of sampling volume 1 . It can be see that spatial distribution is very similar, further justifying the validity of chosen approach to simplify collision modelling.

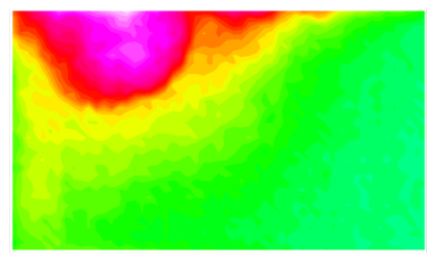

(a)

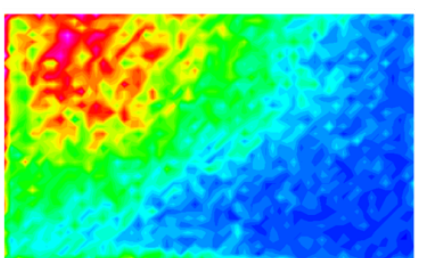

(b)

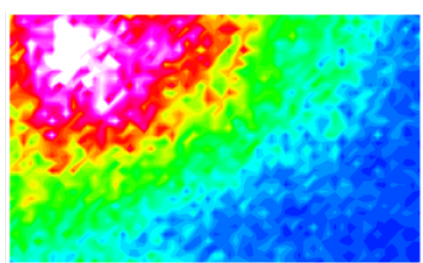

(c)

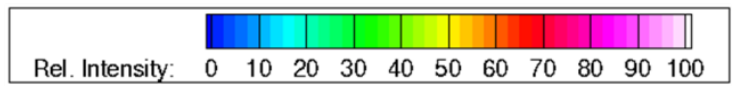

Figure 18: Contamination on the base: Experimental results (a); Numerical results obtained in simulation 3 using original spray (b), and developed spray (c). Numerical results are normalized against the original baseline case shown in Figure 10 (b). 


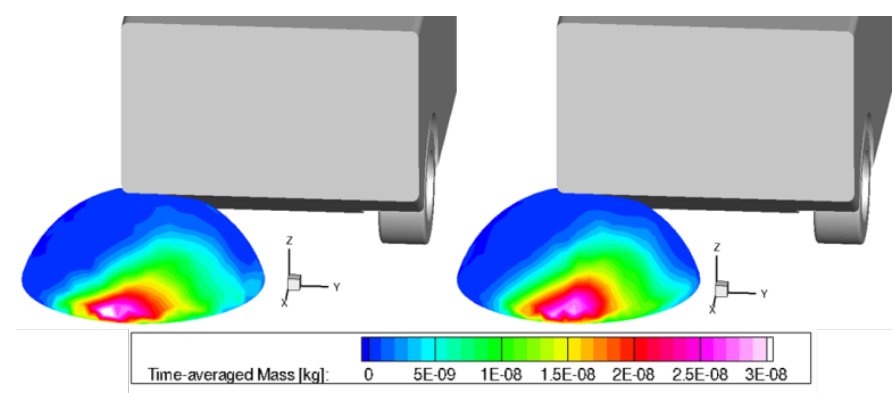

(a)

(b)

Figure 19: Spatial distribution of spray on the surface of sampling volume 1 in simulation 1 cloud 3 (a) and simulation 3 cloud 2 (b).

Results presented suggest that consideration of secondary mechanisms is important in representing the spray and obtaining accurate results. The mechanisms taking place close to the injection point can be modelled separately and a 'developed' spray injected into the full simulation, thus reducing computational cost. However, it should be stressed that the mechanisms studied in this work are problem dependent and the decision of which mechanisms to consider should be always based on factors, such as spray density, initial size and injection velocity, as well as ambient conditions and vehicle speed.

\section{Conclusion}

In this work, the importance of modelling secondary mechanisms that tend to change spray properties, namely particle coalescence, breakup and liquid evaporation have been assessed through the comparison of numerical and experimental results for the generic SUV model. Evaporation tends to remove very small particles from the spray as these tend to evaporate at a high rate, while the coalescence affects particle size distribution by forming large particles out of small ones. In this study, although the break-up mechanism was considered and modelled using the TAB formulation, the spray was too fine for breakup to occur, even when the coalescence of particles was taken into account, and no break-up was detected. The coalescence model used the O'Rourke formulation. This model finds pairs of parcels and uses the probability function to decide whether they will collide or not. The model is computationally expensive as it has to loop over all parcels in the domain. The computational cost depends on the number of parcels, as well as on the outcome of the probability function. The evaporation model used in this work was simplified and considered evaporation at a fixed constant temperature and did not take into account energy conservation. This allows a relatively inexpensive method of including evaporation, without needing to model an additional energy equation, while still predicting important trends.

Three simulations were run in this study. The aim of the first simulation was to assess the influence of each mechanism separately. It used three Lagrangian clouds, tracked concurrently with the continuous phase. The clouds did not influence each other and the back-coupling with the continuous phase was not considered. The first cloud did not use any additional models and the particle size distribution was fixed. The second and third clouds used the evaporation and coalescence models, respectively. As expected, taking the evaporation into account removed very small particles from the spray and significantly reduced the total mass deposited on the base. The coalescence model led to formation of larger particles in the spray, shifting the size distribution towards the bigger particles. Both evaporation and coalescence models were seen to remove the narrow band of high contamination along the edge of the base which is seen in the baseline simulation but not observed in experiment. This region was seen to be due to the deposition of small particles which are removed by the additional spray physics models.

The second simulation was run to see the effect of both mechanisms together. It was found that the coalescence of particles occurs very close to the source, forming bigger particles within a very short time after injection. As a result, the evaporation plays an insignificant role here as it does not affect large particles to the same extent as it does for small particles. In fact, the total mass deposited on the base in this simulation was very similar to the one obtained in the case that considered the coalescence mechanism alone. These results show that the influence of evaporation on vehicle soiling is highly dependent on the other physical processes involved and ambient conditions considered. For this case, the increased mean particle size caused by coalescence causes the evaporation to have little effect. The effect of evaporation is also likely to be small in soiling tests, performed in controlled wind tunnel environments with a saturated atmosphere. But in other circumstances such as on-road full-scale testing, or when the atmospheric humidity is reduced, the particle residence times may increase relative to the evaporation timescale, which may cause more significant differences. On the other hand, road sprays generated by moving vehicles generally contain a diverse range of solid materials, which are not affected by the evaporation processes. Therefore, the effect of spray evaporation for full scale cases and representative contaminant compositions still needs to be further analysed.

It has been shown that the coalescence mechanism can change the particle size distribution in the spray and this should ideally be accounted for, especially when the spray is fine and dense and is injected from a single point in space. This work has shown that for such injection the region in which the coalescence of particles is important is near the source. It is as yet unknown how the coalescence and break-up mechanisms would affect the tyre spray at full scale. The spray ejected by a real rotating tyre is likely to have a different size distribution and density to that seen in this experiment, although more experimental work needs to be done in this area. Consideration of the coalescence mechanism in vehicle soiling studies leads to a substantial increase in computational cost. A possible solution to this has been presented. A simulation is performed with the coalescence model enabled for some time in order to obtain the developed spray size distribution to be found at some distance from injection. This developed size distribution can then be used in full soiling simulations with no coalescence model. In the third simulation we employed this method and used a new spray, obtained by time-averaging the spray used in the second simulation which models coalescence. The sampling and averaging was done in a volume where the spray was shown to have been developed. The new developed spray was injected from the original source and the base contamination was compared against the original simulation and experimental results. Excellent agreement with the experimental results was obtained by using the developed spray. The agreement was better than the one obtained with the original spray and in agreement with the results of the full, multi-physics, simulation.

\section{Acknowledgements}

This work was supported by Jaguar Land Rover and the UK Engineering and Physical Sciences Research Council grant EP/K014102/1 as part of the jointly funded Programme for Simulation Innovation. This research has made use of the HPC-

Page 10 of 12 
Midlands-Plus Tier-2 computing system, supported by the research computing staff of Loughborough University, and funded through EPSRC e-infrastructure grant EP/P020232/1.

\section{Contact Information}

A. Kabanovs

a.kabanovs@lboro.ac.uk

A. Garmory

a.garmory@lboro.ac.uk

M. Passmore

m.passmore@lboro.ac.uk

A. Gaylard

agaylar1@jaguarlandrover.co.uk

\section{References}

[1] A. P. Gaylard, K. Kirwan, and D. A. Lockerby, "Surface contamination of cars: A review," Proc. Inst. Mech. Eng. Part D J. Automob. Eng., vol. 231, no. 9, pp. 1160-1176, Aug. 2017.

[2] M. Kutila, P. Pyykönen, A. Lybeck, P. Niemi, and E. Nordin, "Towards Autonomous Vehicles with Advanced Sensor Solutions,” World J. Eng. Technol., vol. 3, no. 3, pp. 6-17, 2015.

[3] E. D. R. Shearman, E. G. Hoare, and A. Hutton, "Trials of automotive radar and lidar performance in road spray," in IEE Colloquium on Automotive Radar and Navigation Techniques, 1998, vol. 1998.

[4] A. Gaylard and B. Duncan, "Simulation of Rear Glass and Body Side Vehicle Soiling by Road Sprays," SAE Int. J. Passeng. Cars - Mech. Syst., Apr. 2011.

[5] C. Houska and CSI, "Deicing Salt - Recognizing The Corrosion Threat,” pp. 1-11, 2007.

[6] M. Yoshida, A. Yamatani, and H. China, "Prediction of dirt contamination on vehicle body surface," JSAE Rev., vol. 19(3), pp. 257-262, NaN-4304-0, 1998.

[7] A. Kabanovs, M. Varney, A. Garmory, M. A. Passmore, and A. P. Gaylard, "Experimental and Computational Study of Vehicle Soiling on a Generic Hatchback Body,” SAE World Congr., 2016.

[8] J. S. Paschkewitz, "A comparison of dispersion calculations in bluff body wakes using LES and unsteady RANS," Lawrence Livermore National Laboratory, Report No. UCRL-TR-218576, 2006.

[9] J. S. Paschkewitz, "Simulation of spray dispersion in a simplified heavy vehicle wake," Lawrence Livermore National Laboratory, Report No. UCRL-TR-218207, 2006.
[10] J. Jilesen, A. Gaylard, B. Duncan, A. Konstantinov, and J. Wanderer, "Simulation of Rear and Body Side Vehicle Soiling by Road Sprays Using Transient Particle Tracking," SAE Int. J. Passeng. Cars - Mech. Syst., vol. 6, no. 1, pp. 424-435, Apr. 2013.

[11] T. Kuthada and S. Cyr, "Approaches to Vehicle Soiling," 4th FKFS Conf. Prog. Veh. Aerodyn. Therm. Manag. Numer. methods, p. 111-123. Renningen: Expert-Verlag, 2006.

A. Kabanovs, A. Garmory, M. Passmore, and A. Gaylard, "Computational simulations of unsteady flow field and spray impingement on a simplified automotive geometry," J. Wind Eng. Ind. Aerodyn., vol. 171, no. September, pp. 178-195, Dec. 2017.

[13] A. Gaylard, A. Kabanovs, J. Jilesen, K. Kirwan, and D. Lockerby, "Simulation of rear surface contamination for a simple bluff body,” J. Wind Eng. Ind. Aerodyn., vol. 165, pp. 13-22, Jun. 2017.

[14] A. Kabanovs, G. Hodgson, A. Garmory, M. Passmore, and A. Gaylard, "A Parametric Study of Automotive Rear End Geometries on Rear Soiling," SAE Int. J. Passeng. Cars Mech. Syst., vol. 10, no. 2, pp. 553-562, Mar. 2017.

[15] A. Gaylard et al., "Insights into Rear Surface Contamination Using Simulation of Road Spray and Aerodynamics," SAE Int. J. Passeng. Cars - Mech. Syst., vol. 7, no. 2, pp. 673681, Apr. 2014.

[16] L. M. Portela and R. V. A. Oliemans, "Possibilities and Limitations of Computer Simulations of Industrial Turbulent Multiphase Flows,” Eng. Turbul. Model. Exp. 6, pp. 49-63, 2005.

[17] X. Hu et al., "A numerical simulation of wheel spray for simplified vehicle model based on discrete phase method," Adv. Mech. Eng., vol. 7, no. 7, pp. 1-8, 2015.

[18] G. M. Elfstrom and G. L. Rohrauer, "Design and Construction of the UOIT Climatic Wind Tunnel."

[19] A. Wood, M. Passmore, D. Forbes, D. Wood, and A. Gaylard, "Base Pressure and Flow-Field Measurements on a Generic SUV Model,” SAE Int. J. Passeng. Cars - Mech. Syst., vol. 8, no. 1, pp. 233-241, Apr. 2015.

[20] G. Johl, M. Passmore, and P. Render, "Design methodology and performance of an indraft wind tunnel," Aeronaut. J., vol. 108, no. 1087, pp. 465-473, 2004.

[21] OpenFOAM, “v.2.4.0.”

[22] D. B. Spalding, "A Single Formula for the 'Law of the Wall,” J. Appl. Mech., vol. 28, no. 3, pp. 455-458, 1961.

[23] M. L. Shur, P. R. Spalart, M. K. Strelets, and A. K. Travin, "A hybrid RANS-LES approach with delayed-DES and wall-modelled LES capabilities,” Int. J. Heat Fluid Flow, vol. 29, no. 6, pp. 1638-1649, Dec. 2008. 
[24] P. Spalart and S. Allmaras, "A one-equation turbulence model for aerodynamic flows," in La Recherche Aerospatiale, 1994, vol. 1, pp. 5-21.

[25] D. Migdal and V. D. Agosta, “A Source Flow Model for Continuum Gas-Particle Flow,” J. Appl. Mech., vol. 34, no. 4, pp. 860-865, 1967.

[26] A. D. Gosman and E. Ioannides, "Aspects of Computer Simulation of Liquid-Fueled Combustors,” J. Energy, vol. 7, no. 6, pp. 482-490, Nov. 1983.

[27] D. B. Spalding, "The combustion of liquid fuel, 4th Symposium (Int.) on Combustion,” 1953, pp. 847-864.

[28] E. Ranz, W. and R. Marshall, W., "Evaporation From Drops,” in Chem. Eng. Prog, 48, 1952, pp. 141-146.

[29] G. J. Jenkins, M. C. Perry, and M. J. Prior, The climate of the UK and recent trends. 2009.

[30] P. J. O’Rourke, “Collective Drop Effects on Vaporizing Liquid Sprays. PhD Dissertation, Department of Mechanical and Aerospace Engineering, Princeton University,” 1981.

[31] D. P. Schmidt and C. J. Rutland, "Reducing Grid Dependency in Droplet Collision Modeling,” J. Eng. Gas Turbines Power, vol. 126, no. 2, p. 227, 2004.

[32] Z. Hui-ya, Z. Yu-sheng, X. Bo, and M. Chun-lan, "Extension of O' Rourke Droplet Collision Model : Application to Diesel Spray of Single-hole Injector," Engineering, no. 724, 2006.

[33] Q. Li, T. M. Cai, G. Q. He, and C. B. Hu, "Droplet collision and coalescence model,” Appl. Math. Mech. (English Ed., vol. 27, no. 1, pp. 67-73, 2006.

[34] P. J. O’Rourke and A. A. Amsden, “The Tab Method for Numerical Calculation of Spray Droplet Breakup,” 1987. 